DOI: $10.19177 /$ reen.v12e32019203-228

\title{
LEALDADE DE CLIENTES NO MERCADO DE SEGUROS DE AUTOMÓVEIS
}

\section{CUSTOMER LOYALTY IN THE AUTO INSURANCE MARKET}

\section{LEALTAD DE CLIENTES EN EL MERCADO DE SEGUROS DE AUTOMÓVILES}

\author{
Alexandre Yutaca Ikenami \\ Mestre em administração pela Pontifícia Universidade Católica (PUC-Rio) \\ Professor na PUC-Rio \\ Endereço: PUC, R. Marquês de São Vicente, n.225, Gávea, CEP: 22451-900. Rio de Janeiro, RJ, Brasil \\ Telefone: (21) 2138-9337 \\ E-mails: alexandre.ikenami@outlook.com
}

\section{Jorge Brantes Ferreira}

Doutor em administração pelo Instituto de Pós-Graduação e Pesquisa em Administração da Universidade Federal do Rio de Janeiro (COPPEAD-UFRJ)

Professor na PUC-Rio

Endereço: PUC, R. Marquês de São Vicente, n.225, Gávea, CEP: 22451-900. Rio de Janeiro, RJ, Brasil Telefone: (21) 2138-9337

E-mail: jorgebf@gmail.com

\section{Angilberto Sabino de Freitas}

Doutor em administração pela PUC-Rio

Professor na Universidade do Grande Rio (UNIGRANRIO)

Endereço: UNIGRANRIO, R. da Lapa, n. 86, Centro, CEP: 20021-180. Rio de Janeiro, RJ, Brasil

Telefone: (21) 98003-8338

E-mail: angilberto.freitas@gmail.com

\section{Luciana Sousa Coelho Marson}

Mestra em administração pela Universidade Federal Fluminense (UFF)

Professora na UNIGRANRIO

Endereço: UNIGRANRIO, R. da Lapa, n. 86, Centro, CEP: 20021-180. Rio de Janeiro, RJ, Brasil

Telefone: (21) 98759-0656

E-mail: luciana.marson@globo.com

\section{Fernanda Leão Ramos}

Mestra em administração pela PUC-Rio

Professora na PUC-Rio

Endereço: PUC, R. Marquês de São Vicente, n.225, Gávea, CEP: 22451-900. Rio de Janeiro, RJ, Brasil Telefone: (21) 2138-9337

E-mail: leaoramos@gmail.com

Artigo recebido em 20/04/2019. Revisado por pares em 18/07/2019. Reformulado em 10/11/2019. Recomendado para publicação em 01/12/2019. Publicado em 23/12/2019. Avaliado pelo Sistema double blind review. 


\section{RESUMO}

O objetivo deste estudo é avaliar como a satisfação, o custo de mudança, a atratividade das alternativas e a confiança afetam a formação da lealdade do cliente no seguro de automóvel. Para verificar as relações sugeridas no modelo conceitual proposto, foi conduzida uma survey online com indivíduos que contrataram uma apólice de seguros de automóvel durante o prazo máximo de dois anos anteriores ao momento da pesquisa. Os dados levantados foram analisados por meio de equações estruturais e os resultados obtidos sugerem que satisfação afeta positivamente a confiança e a lealdade do consumidor no mercado de seguro de automóvel.

Palavras-chave: Lealdade do consumidor; Seguros de automóvel; Confiança do cliente; Satisfação do cliente; SEM.

\section{ABSTRACT}

The objective of this study is to evaluate how satisfaction, cost of change, attractiveness of alternatives and trust affect customer loyalty in auto insurance. To verify the suggested relationships in the proposed conceptual model, an online survey was conducted with individuals who contracted an automobile insurance policy for a maximum period of two years prior to the time of the survey. The data collected were analyzed through structural equations and the results obtained suggest that satisfaction positively affects consumer confidence and loyalty in the auto insurance market.

Keywords: Consumer Loyalty; Auto Insurance; Customer confidence; Customer satisfaction; SEM.

\section{RESUMO}

El objetivo de este estudio es evaluar cómo la satisfacción, el costo de cambio, el atractivo de las alternativas y la confianza afectan a la formación de la lealtad del cliente en el seguro de automóvil. Para verificar las relaciones sugeridas en el modelo conceptual propuesto, se llevó a cabo una encuesta en línea con individuos que contrataron una póliza de seguros de automóvil durante el plazo máximo de dos años anteriores al momento de la investigación. Los datos levantados se analizaron a través de ecuaciones estructurales y los resultados obtenidos sugieren que la satisfacción afecta positivamente la confianza y la lealtad del consumidor en el mercado del seguro de automóvil.

Palabras clave: Lealtad del consumidor; Seguros de automóvil; Confianza del cliente; Satisfacción del cliente; SEM. 


\section{INTRODUÇÃo}

A atividade seguradora possui uma participação relevante na economia nacional. De acordo com dados da Superintendência de Seguros Privados, SUSEP, o mercado securitário movimentou R\$ 360 bilhões de reais em 2016, valores que representam 3,8\% do PIB brasileiro e, mesmo com o contexto macroeconômico desfavorável, apresentou um crescimento de 4\% na comparação com 2015.

Dentro deste mercado, o seguro de automóvel apresentou um volume total de 3 bilhões de reais de prêmio emitido, com cerca de 5 milhões de apólices contratadas em 2016 (CNSEG, 2016). Atualmente, mais de 10 companhias trabalham com seguros de automóvel. Essa concorrência diminui a margem de lucros, dificulta o potencial de crescimento e torna o segurado mais exigente. Para enfrentar os desafios desse mercado, as seguradoras investem em ações para tentar entender melhor os seus clientes, para assim desenvolverem estratégias com o intuito de fideliza-los. Nesse sentido, compreender como se devolve a lealdade entre cliente e seguradora pode ser um diferencial para a manutenção desses segurados por um longo tempo, assim como para captação de novos clientes, aumentando seu crescimento no mercado.

Dentro deste contexto, o objetivo deste estudo é investigar que antecedentes impactam na lealdade do consumidor de seguros de automóvel e avaliar as relações entre esses antecedentes e a lealdade. O estudo é delimitado a segurados que contrataram uma apólice de seguros de automóvel durante o período máximo de dois anos anteriores ao momento da pesquisa.

\section{REFERENCIAL TEÓRICO}

A seguir é apresentada a literatura relacionado ao objeto de pesquisa deste estudo. Primeiro, apresenta-se a literatura referente à lealdade e os construtos que a impactam. Em seguida é proposto um modelo e as hipóteses para avaliar a lealdade do consumidor de seguros de automóvel.

\subsection{LEALDADE}


O conceito da lealdade tem sido investigado por meio três abordagens distintas: comportamental, atitudinal e integrada (DAY, 1969; JACOBY; KYNER, 1973; DICK; BASU, 1994; GREMLER; BROWN, 1999; OLIVER 1997, 1999, HART et al. 1999).

A lealdade comportamental se baseia na observação do comportamento de recompra ao longo do tempo (UNCLES et al., 2003). Essa abordagem analisa apenas o histórico de compras do cliente para mensurar sua lealdade com a marca (CUNNINGHAM, 1956; TUCKER; 1964; KAHN, et al., 1986). É vantajoso o uso de medidas comportamentais quando a escolha, de modo geral, faz parte da rotina do consumidor, em que existe um baixo grau de envolvimento com o produto/serviço comprado. O cliente não enxerga a empresa dentro do seu conjunto de valores simbólicos (UNCLES et al., 2003).

Por outro lado, alguns autores criticam medidas de lealdade baseadas apenas em critérios comportamentais, por refletirem uma visão estreita do relacionamento entre empresa e clientes (OLIVER, 1997). A repetição de compras pode não representar uma preferência, sendo apenas fruto de uma inércia, de um alto custo de troca ou até mesmo uma indiferença em relação aos demais produtos ou serviços ofertados pelos concorrentes.

Assim, Jacoby e Kyner (1973) elaboraram um modelo de lealdade que engloba tanto componentes comportamentais como os atitudinais. O autores definem lealdade em função de seis condições necessárias e coletivamente suficientes: (1) lealdade à marca é a ação comportamental; (2) intencional; (3) expressa no tempo; (4) por uma unidade de compra; (5) em relação a um conjunto de marcas alternativas e; (6) em função de processos psicológicos. Esses componentes proporcionam ao cliente uma avaliação mais criteriosa na escolha das alternativas, e consequentemente na tomada de decisão, o que o tornará mais leal na formação de seu comprometimento em relação à marca.

Oliver (1997) também considera aspectos comportamentais e atitudinais e apresenta uma visão integrada de lealdade que busca explorar aspectos cognitivos, afetivos e comportamentais, enfatizando os sentimentos e emoções dos consumidores, além dos hábitos de compra. O autor define a lealdade como um comprometimento em comprar novamente um mesmo produto ou serviço de uma determinada marca no futuro, apesar das 
influências situacionais e dos esforços da concorrência que buscam mudar seu comportamento de consumo (OLIVER, 1997).

\subsection{SATISFAÇÃO}

A satisfação é definida por Oliver $(1997,1999)$ como a percepção do consumidor de que um desejo, necessidade ou objetivo foi satisfeito de forma suficiente por meio de uma experiência de consumo. POde-se dizer que a satisfação do consumidor é um tipo de avaliação entre a expectativa e percepção de desempenho de um determinado produto ou serviço (CHEN; WANG, 2009; PEREIRA et al., 2016). A resposta do consumidor a essas comparações entre desempenho e expectativa pode ser entendida então como a satisfação do cliente. Conforme descreve Oliver (1997), satisfação é a resposta-realizada do consumidor. É um julgamento de que um produto/serviço oferece um nível prazeroso de consumo. Fornell (1992) também define satisfação como uma avaliação geral do desempenho com base em todas as experiências anteriores.

Os autores Garbarino e Johnson (1999) afirmam que existem dois tipos de satisfação. A primeira seria a satisfação cumulativa, que é um conjunto de avaliações das experiências de consumo feitas ao longo do tempo; a outra seria a satisfação de transações específicas, que é entendida por uma avaliação feita imediatamente após o consumo sendo realizado então um julgamento imediato ou uma reação afetiva a mais recente experiência com a marca.

Se as experiências positivas do cliente excederem suas expectativas, provavelmente os clientes estarão mais satisfeitos. Assim, a satisfação do cliente pode ser definida como uma avaliação de que sua experiência foi pelo menos tão boa quanto supostamente era esperado (HUNT, 1977).

\subsection{CUSTO DE TROCA}

Porter (1980) descreve que o custo de troca (ou custo de mudanças) é a percepção da magnitude ou grandeza dos custos - financeiros e não financeiros - adicionais requeridos para terminar o relacionamento corrente e optar por uma alternativa concorrente. Para os autores Morgan e Hunt (1994), os custos de troca são apontados como um fator que 
contribui para a manutenção do relacionamento entre a empresa e o consumidor. Todavia, para esses autores, os custos de troca possuem natureza meramente econômica.

Outros autores, como Sharma (2003), afirmam que os custos de troca podem apresentar aspectos psicológicos e emocionais, ou componentes de custos ligados a aspectos de relacionamento (GHAZALI et al., 2016). Um exemplo é o vínculo de confiança construído ao longo do tempo com uma empresa, que cria uma barreira que não é financeira. Assim, esse efeito psicológico atua como um custo, mesmo que o desempenho do serviço ou do produto seja menor que o satisfatório. A troca de fornecedor envolveria também outros esforços não monetários, como tempo e reflexão.

Portanto, para esta pesquisa, seguindo sugestão de Wang et al. (2001) e Nesset e Helgesen (2014), a definição de custo de troca é a percepção do tempo, dinheiro e esforço despendido necessário para que o consumidor efetue a mudança de uma empresa para uma outra. Esse custo pode envolver também um comprometimento afetivo, que pode representar uma barreira psicológica e emocional à mudança.

\subsection{ATRATIVIDADE DAS ALTERNATIVAS}

A relevância de uma alternativa concorrente tem sido muito pesquisada em estudos sobre a tomada de decisão do consumidor e sobre relações interpessoais (HUNG, et al., 2006). A teoria do arrependimento (BELL, 1982; LOOMES; SUGDEN, 1982) identificou que indivíduos podem sentir arrependimento ou alegria diante do resultado de uma decisão. A pessoa se arrepende se o resultado obtido foi pior que a alternativa concorrente e se sente feliz quando atinge um resultado melhor. A teoria discute basicamente a probabilidade associada à escolha que pode ser de conforto ou arrependimento, fazendo com que o valor da decisão escolhida dependa dos itens simultaneamente excluídos. Se as opções preteridas forem de grande atratividade, o valor da escolha e as probabilidades de arrependimento se intensificam.

\subsection{CONFIANÇA}

Para Morgan e Hunt (1994), o conceito de confiança pode ser definido como a necessidade ou vontade de poder contar com um outro indivíduo parceiro no qual se 
deposita a confiança. Mayer et al. (1995) definem confiança como a disposição de um indivíduo a ficar exposto ou vulnerável às ações de outro com base na expectativa de que a outra parte cumprirá uma ação considerada importante.

O cliente avalia o desempenho de uma empresa após uma experiência de consumo e o seu resultado pode reforçar ou diminuir a sua confiança. Será considerada confiável uma empresa que possuir uma elevada percepção das três dimensões: benevolência, competência e integridade (MAYER et al., 1995).

\subsection{MODELO CONCEITUAL E HIPÓTESES}

\subsubsection{Satisfação e Lealdade}

$\mathrm{Na}$ literatura, a satisfação é o antecedente da lealdade mais estudado e, na grande maioria, as pesquisas apontam uma relação positiva significante entre satisfação e lealdade (VALVI; FRAGKOS, 2012). Clientes satisfeitos possuem uma maior probabilidade de recompra, são clientes com uma menor sensibilidade aos preços e usualmente possuem um maior envolvimento com a marca através de recomendações boca-a-boca positivas (CHEN; WANG, 2009). Entretanto, a satisfação não se converte obrigatoriamente em lealdade, apesar de consumidores leais serem usualmente satisfeitos (OLIVER, 1999).

Particularmente, a satisfação é considerada um antecedente importante da lealdade na indústria de serviços (HAGHIGHI et al., 2012; HAU; THUY 2012 e SULTAN; WONG 2013). Um estudo realizado por Sultam e Wong (2013) confirmou que clientes satisfeitos desenvolvem preferência por uma determinada seguradora na qual oferece serviços satisfatórios. O nível percebido de satisfação do cliente explicou uma quantidade considerável de variação de preferência de marca. Picón et al. (2014) afirmam que uma das formas de se elevar a lealdade do cliente é por meio do aumento de sua satisfação. Assim, considera-se a hipótese:

H1. A satisfação possui um efeito direto e positivo sobre a lealdade.

\subsubsection{Satisfação, Custo de troca e Lealdade}


De acordo com Oliver (1999), a satisfação é um passo necessário no processo de construção da lealdade. Entretanto, esta variável se torna menos significativa quando outros mecanismos (tais como vínculos sociais e pessoais, a existência de obrigações contratuais, etc.) influenciam essa lealdade. Todos esses fatores criam custos de mudança que fortalecem a retenção de clientes. Por outro lado, estudos mostram como o custo de troca desempenha um papel importante no que diz respeito à satisfação ao explicar a lealdade (PATTERSON; SMITH, 2003; TSAl et al., 2006).

Corroborando com esses aspectos, Ngobo (2004) defende a visão de que a satisfação do cliente determina o nível da percepção do custo de troca. $O$ autor acredita que a satisfação do cliente tem um efeito positivo sobre os custos de troca, aumentando assim a percepção de desvantagem do cliente na troca de um fornecedor. Por isso, quanto maior a satisfação geral, maiores serão os custos de troca ou maior será a expectativa de perda numa eventual mudança de marca feita pelo cliente. Ou seja, o consumidor percebe maiores dificuldades de romper o relacionamento corrente ou associa maior inutilidade na troca de fornecedores quando ele possui um alto nível de satisfação com sua empresa atual (NGOBO, 2004). Através da criação de custos de mudança, as empresas podem desencorajar os clientes a mudarem para um concorrente. (BARROSO; PICÓN, 2012).

A natureza desses custos (relacional, monetária, psicológica ou associada ao tempo que o processo de mudança assume) pode ser muito diferente dependendo do setor ou da indústria. Em seu estudo sobre a lealdade no mercado de seguros, Picón et al. (2014) identificaram que a criação do custo de troca é uma das formas de se elevar a lealdade. 0 cliente com um alto custo percebido de troca tende a manter o relacionamento com a seguradora atual. Custos de troca afetam a lealdade, dificultando a mudança para outro fornecedor. Portanto, esta pesquisa investiga as hipóteses:

H2. A satisfação possui um efeito direto e positivo sobre o custo de troca

H3. O custo de troca possui um efeito direto e positivo sobre a lealdade.

\subsubsection{Satisfação, Atratividade das Alternativas e Lealdade}


A atratividade das alternativas aparece na literatura como um fator que motiva e favorece a mudança para uma marca concorrente. Caso contrário, em um mercado com baixa concorrência e sem muita oferta de produtos similares, a baixa atratividade das alternativas aumenta a lealdade à marca habitual. A definição do nível da atratividade das alternativas disponível é a probabilidade do cliente obter satisfação através de uma marca alternativa (BANSAL et al., 2005). Portanto, aqueles clientes satisfeitos com sua marca habitual perceberão que as outras ofertas que existem no mercado são menos atraentes. Os clientes avaliarão comparativamente, por meio de uma análise custo-benefício, de tal forma que se o cliente perceber que o provedor existente é superior, o benefício da mudança será menor.

No entanto, a empresa não controla esta variável em algumas situações, uma vez que o cliente pode decidir permanecer na relação dada a estrutura de mercado, ou porque, embora existam ofertas alternativas, essas ofertas estão fora da gama de opções do cliente. Assim, se os clientes não estão cientes da atratividade das alternativas, ou simplesmente não percebem qualquer oferta alternativa como sendo mais atraente do que o provedor atual, eles são mais propensos a permanecer no relacionamento (PATTERSON; SMITH, 2003).

Portanto, as empresas que tentam se diferenciar de seus concorrentes, oferecendo preços mais competitivos, melhor qualidade de serviço, melhor atendimento ao cliente e assim por diante - aumentando o nível de satisfação - estão trabalhando para a redução da atratividade das alternativas (ANTÓN et al., 2007) e, portanto, estimulam a lealdade dos clientes. Assim, duas hipóteses são propostas:

H4. A satisfação possui um efeito direto e negativo sobre a atratividade das alternativas.

H5. A atratividade das alternativas possui um efeito direto e negativo sobre a lealdade.

\subsubsection{Satisfação, Confiança e Lealdade}


A confiança desempenha um papel fundamental na construção de um relacionamento de longo prazo entre a marca e o cliente. Chaudhuri e Holbrook (2001) definem a confiança na marca como a disposição do consumidor de confiar na capacidade da marca para desempenhar sua função declarada. Em geral, a confiança envolve dois parceiros numa relação de troca. A confiança na marca se baseia na percepção de que a empresa é confiável e responsável pelo interesse e pelo bem-estar do usuário.

Normalmente, um cliente insatisfeito gera um boca-a-boca negativo. Por outro lado, um cliente satisfeito pode gerar um boca-a-boca positivo e assim um aumento no nível de confiança da marca. Confiança na marca leva à lealdade, porque a confiança cria relações de troca que são valorizadas. Uma consequência importante da confiança é um relacionamento duradouro (AURIER; N'GOALA, 2010). Pesquisadores que examinaram empiricamente a relação entre confiança e lealdade identificaram que a confiança é um fator determinante da fidelidade à marca (MORGAN; HUNT, 1994; CHAUDHURI; HOLBROOK, 2001; SIRDESHMUKH et al., 2002; AURIER; N'GOALA, 2010). Assim, são propostas as hipóteses:

H6. A satisfação possui um efeito direto e positivo sobre a confiança.

H7. A confiança possui um efeito direto e positivo sobre a lealdade.

Para verificar as hipóteses, foi proposto o modelo da Figura 1, desenvolvido a partir de uma adaptação do modelo proposto por Picón et al. (2014) com a inclusão do construto confiança, utilizado no modelo de Aurier e N'Goala (2010). 
Figura 1 - Modelo Conceitual Proposto

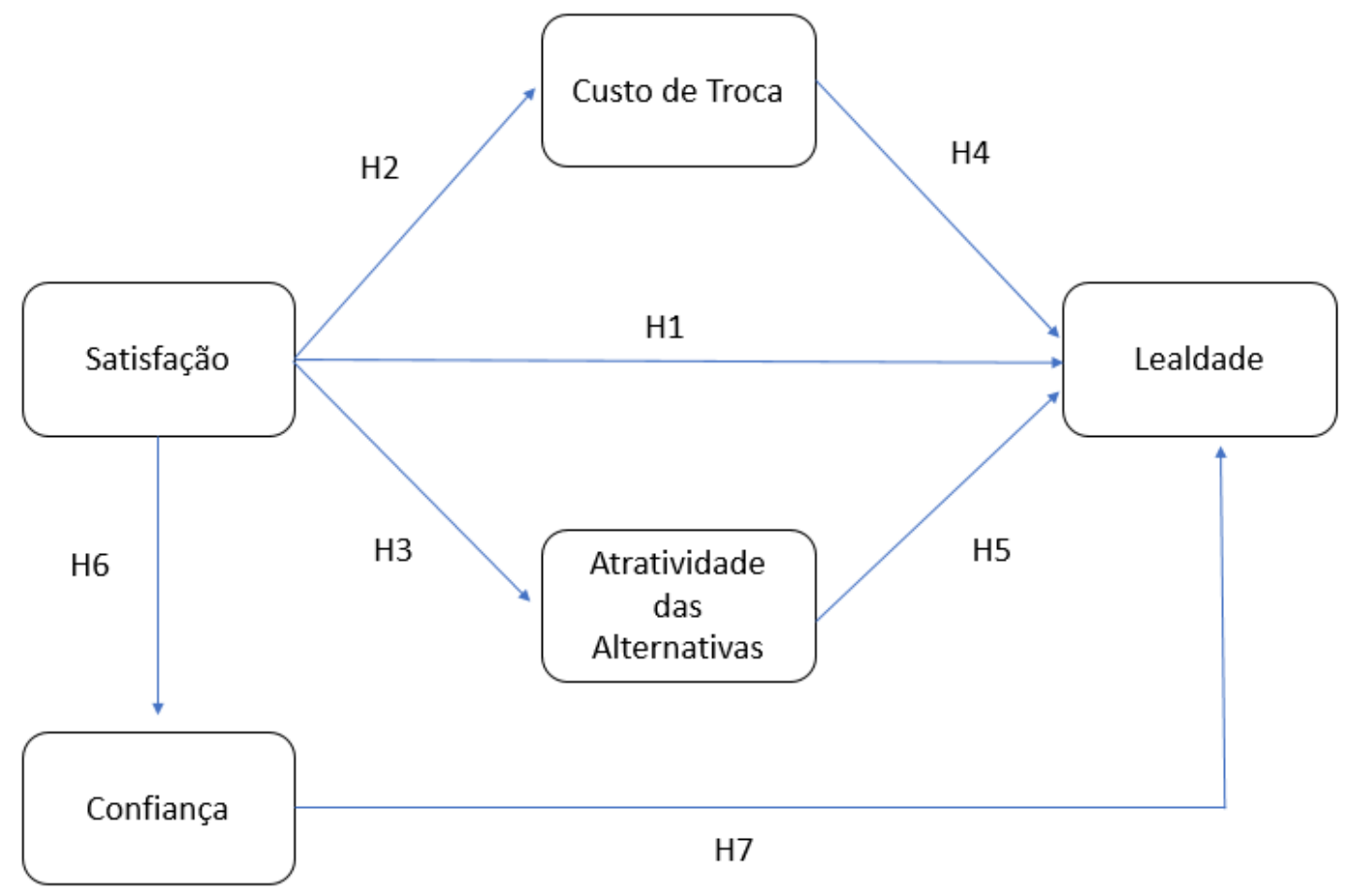

Fonte: Adaptado de Picón et al. (2014) e Aurier e N’Goala (2010), 2019.

\section{METODOLOGIA}

Com o objetivo de testar as hipóteses propostas no estudo, foi realizada uma crosssectional survey (PARASURAMAN et al., 2006), por meio de questionários auto administrados via internet (AAKER et al., 2006), com uma amostra não probabilística da população de interesse.

A coleta de dados se deu por meio de questionários elaborados na plataforma do site Qualtric.com. O link de acesso do questionário on-line foi distribuído via aplicativo de mensagem para smartphones (Whatsapp) e por meio de mensagens em redes sociais (Facebook e Linkedin) para cerca de 500 indivíduos da rede do pesquisador. Como o perfil do respondente era razoavelmente específico, não foi solicitado repassar o link da pesquisa para evitar respondentes fora do público alvo.

\subsection{OPERACIONALIZAÇÃO E DEFINIÇÃO DAS VARIÁVEIS}

Este estudo utilizou-se de escalas já elaboradas e testadas pela literatura para a medição dos construtos envolvidos. Para tanto, foram utilizadas as seguintes escalas: 
- Satisfação - Escala de 7 itens adaptada de Picón et al. (2014).

- Custo de Troca - Escala de 15 itens adaptada de Picón et al. (2014).

- Atratividade das Alternativas - Escala de 4 itens adaptada de Picón et al. (2014).

- Lealdade - Escala de 8 itens adaptada de Picón et al. (2014).

- Confiança - Escala de 4 itens adaptada de Aurier e N’Goala (2010).

Originalmente, as escalas utilizadas neste estudo foram desenvolvidas na língua inglesa, sendo necessária a tradução e adaptação de cada escala para o português por profissionais fluentes, com etapas de tradução e retradução sendo empregadas para garantir que as escalas em português se aproximassem o máximo possível das originais (SPERBER, 2004).

Para validação da tradução e da adequação do questionário, foi realizado um préteste do questionário com uma pequena amostra do público de interesse para avaliar a compreensão dos respondentes sobre esta primeira versão do questionário. Os resultados obtidos com esse pré-teste inicial serviram para refinar o questionário e elaborar uma nova versão. Essa versão ainda passou por um pré-teste final, em que foi verificado se algum último ajuste era necessário. Com os resultados desse último pré-teste, foi elaborado o instrumento de pesquisa final, com um total de 38 itens medidos por meio de escalas Likert de cinco pontos, variando entre "discordo totalmente" e "concordo totalmente". Além dessas dimensões, o questionário também apresentava itens para avaliar o perfil dos respondentes.

A população de interesse é formada por adultos brasileiros que possuem ou já possuíram seguro de automóvel contratado nos últimos 24 meses. A amostra estudada foi composta por brasileiros predominantemente residentes do Rio de Janeiro, que utilizam carro com seguro de automóvel contratado durante o prazo máximo de dois anos anteriores ao momento da pesquisa.

\subsection{COLETA DE DADOS}


Os dados foram coletados por meio de uma survey online. O processo de coleta de dados foi realizado ao longo do mês de fevereiro de 2017 e a participação dos respondentes foi voluntária e confidencial. O objetivo em relação aos respondentes era que fossem adultos maiores de 18 anos e tivessem algum conhecimento e/ou experiência de uso com seguro de automóvel, por isso foram considerados apenas respondentes que utilizassem carro com seguro de automóvel contratado no período de até dois anos ao momento da pesquisa, resultando em uma amostra final com 230 respostas válidas. O questionário era auto administrado e os participantes foram voluntários interessados no tema.

A amostra apresentou uma concentração de respondentes do sexo masculino (80\%), com apenas $20 \%$ dos respondentes sendo do sexo feminino. Com relação à faixa etária dos respondentes, $48,70 \%$ estão na faixa entre 30 a 50 anos, seguidos de $29,57 \%$ que afirmaram estar na faixa entre 18 a 30 anos e por fim 21,74\% apontaram ter mais de 50 anos. Com relação à renda familiar, a maior parte, com $46 \%$ dos participantes, estimou uma renda acima de 10 mil reais, 34\% estimaram uma renda entre 5 a 10 mil e $20 \%$ estimaram renda na faixa entre mil a 5 mil reais. Nenhum respondente afirmou ter uma renda familiar abaixo de mil reais.

Do total de 230 participantes, 153 participam da negociação e contratação do seguro de automóvel, representando $66,5 \%$ do total da amostra, enquanto 77 dos respondentes (34,5\% do total) apenas utilizam o veículo e não participam do processo de contratação do seguro de automóvel.

\section{ANÁLISE DOS RESULTADOS}

Esta seção tem por objetivo apresentar os resultados, primeiramente descrevendo os resultados do modelo de mensuração e em seguida os resultados do modelo estrutural, em que se apresenta o teste das hipóteses propostas.

\subsection{AVALIAÇÃO DO MODELO DE MENSURAÇÃO}

No presente estudo, por meio do uso do software AMOS 20, foi realizada uma análise fatorial confirmatória (CFA) para analisar a validade, a unidimensionalidade e a 
confiabilidade das escalas utilizadas no modelo de mensuração para verificar o quanto cada item medido no modelo de mensuração se relaciona com cada construto em particular.

Inicialmente, com o modelo utilizando todos os 38 indicadores medidos, os índices de ajuste encontrados ficaram abaixo dos valores recomendados pela literatura. Assim, por meio da análise da matriz de covariância dos resíduos padronizados da CFA, foram identificados os itens que pudessem estar contribuindo para o fraco ajuste do modelo. Dessa forma, foram eliminados 11 itens: 2 itens da escala de satisfação; 5 da escala de custo de troca; 1 da escala de atratividade das alternativas; 2 da escala de lealdade; e 1 da escala de confiança.

Por fim, o modelo de mensuração final, com 27 indicadores, apresentou bons índices de ajuste $(\mathrm{SRMR}=0,0654$; RMSEA=0,087 com C.I. de 0,080 até 0,094; $\mathrm{CFI}=0,868 ; \mathrm{IFI}=0,869$; $T L I=0,852 ; x^{2}=858,052$, d.f. $\left.=314, p<0,001, x^{2} / d . f .=2,733\right)$, o que representou uma melhora significativa em relação ao modelo inicial. Em conjunto, esses índices apontam que os dados se ajustam de maneira satisfatória ao modelo proposto (HU; BENTLER, 1999).

Com relação a validade de face, essa foi garantida durante o desenvolvimento do instrumento de pesquisa por meio da escolha de escalas utilizadas anteriormente na literatura, da tradução criteriosa dessas escalas do inglês para o português, da avaliação de cada item por pesquisadores experientes e dos pré-testes conduzidos.

Com relação à validade nomológica, pode-se concluir que os construtos utilizados apresentam a validade nomológica, uma vez que todas as correlações são consistentes com a teoria estudada e estando na direção apontada pela literatura. As correlações obtidas estão dispostas na tabela 1, acima da diagonal principal.

Em relação à confiabilidade composta, todas as escalas utilizadas atendem aos níveis mínimos de confiabilidade considerados adequados pela literatura, apresentando valores acima de 0,7 (FORNELL; LARCKER, 1981). Também foi calculada a variância extraída média para cada construto (average variance extracted - AVE) para avaliar a validade convergente. A validade convergente é considerada adequada quando estimativas de AVE são maiores do que 0,50 (FORNELL; LARCKER, 1981). Os resultados obtidos indicam a confirmação da 
validade convergente das escalas utilizadas, pois todos os valores AVE calculados estão entre 0,56 e 0,72 (acima do nível recomendado de 0,5).

Por fim, verificou-se a validade discriminante, comparando a variância extraída média (AVE) com a variância compartilhada (o quadrado do coeficiente de correlação) entre todos os pares de construtos, sendo possível verificar que as variâncias compartilhadas são inferiores à AVE, indicando validade discriminante adequada (tabela 1, com a AVE na diagonal principal e as variâncias compartilhadas abaixo da diagonal principal).

Tabela 1- Correlações, AVE e Matriz de Validade Discriminante

\begin{tabular}{|l|l|l|l|l|l|}
\cline { 2 - 7 } \multicolumn{1}{c|}{} & \multicolumn{1}{c|}{$\mathbf{1}$} & \multicolumn{1}{|c|}{$\mathbf{2}$} & $\mathbf{3}$ & $\mathbf{4}$ & \multicolumn{1}{c|}{$\mathbf{5}$} \\
\hline Satisfação (1) & 0,68 & $-0,054$ & 0,320 & 0,570 & 0,571 \\
\hline Custo de Troca (2) & 0,003 & 0,56 & 0,243 & 0,510 & 0,225 \\
\hline Atratividade das Alternativas (3) & 0,102 & 0,059 & 0,70 & 0,034 & 0,214 \\
\hline Confiança (4) & 0,325 & 0,260 & 0,001 & 0,72 & 0,657 \\
\hline Lealdade (5) & 0,326 & 0,050 & 0,045 & 0,431 & 0,67 \\
\hline
\end{tabular}

Nota: A diagonal principal contém os valores para AVE, acima da diagonal estão as correlações e abaixo estão as variâncias compartilhadas.

Fonte: Elaborado pelos autores, 2019.

\subsection{ANÁLISE DO MODELO ESTRUTURAL}

A investigação das relações propostas entre os construtos latentes foi realizada por meio da estimação do modelo estrutural, com o uso do software AMOS 20. Todos os índices de ajuste indicaram um bom ajuste do modelo aos dados. A razão $\chi 2 /$ d.f. foi de 2,73 , inferior ao valor de 3,0 sugerido por Byrne (2010). O SRMR foi de 0,081, enquanto o RMSEA foi de 0,088 (C. I. de 0,081 até 0,095$)$, ambos próximos de 0,08 , conforme recomendado pela literatura ( $\mathrm{HU}$; BENTLER, 1999). Os índices de ajuste incrementais ficaram dentro do patamar de 0,90, com um CFI de 0,87 , um TLI de 0,85 e um IFI de 0,87, ligeiramente abaixo do recomendado, mas ainda assim dentro do limite aceitável para verificação das hipóteses (HU; BENTLER, 1999).

Tabela 2 - Coeficientes Estimados, Hipóteses e Significâncias para o Modelo Proposto

\begin{tabular}{l|llll}
\hline \multicolumn{1}{c|}{ Relação Proposta } & \multicolumn{1}{c}{$\begin{array}{c}\text { Coeficiente } \\
\text { Padronizado }\end{array}$} & \multicolumn{1}{c}{ p-value } & \multicolumn{1}{c}{$\begin{array}{c}\text { Hipótese } \\
\text { Verificada }\end{array}$} \\
\hline H1: Satisfação $\rightarrow$ Lealdade & 0,247 & $<0,001$ & Sim \\
H2: Satisfação $\rightarrow$ Custo de Troca & $-0,054$ & 0,447 & Não \\
H3: Satisfação $\rightarrow$ Atratividade das Alternativas & $-0,314$ & $<0,001$ & Sim \\
H4: Custo de Troca $\rightarrow$ Lealdade & 0,252 & $<0,001$ & Sim \\
H5: Atratividade das Alternativas $\rightarrow$ Lealdade & $-0,167$ & 0,007 & Sim
\end{tabular}




\begin{tabular}{l|l|l|l}
\hline \multicolumn{1}{c|}{ Relação Proposta } & \multicolumn{1}{c|}{$\begin{array}{c}\text { Coeficiente } \\
\text { Padronizado }\end{array}$} & $\begin{array}{c}\text { Hipótese } \\
\text { Verificada }\end{array}$ \\
\hline H6: Satisfação $\rightarrow$ Confiança & 0,566 & $<0,001$ & Sim \\
H7: Confiança $\rightarrow$ Lealdade & 0,490 & $<0,001$ & $\operatorname{Sim}$ \\
\hline
\end{tabular}

Fonte: Elaborado pelos autores, 2019.

A verificação das hipóteses foi realizada por meio da análise da magnitude, direção e significância dos coeficientes padronizados estimados pelo modelo estrutural (BYRNE, 2010). Os coeficientes estimados para o modelo, assim como as hipóteses e significâncias associadas, podem ser observados na tabela 2 e na figura 2. Pode-se observar que foi obtido suporte confirmatório para seis das sete hipóteses apresentadas. Dessas seis, cinco são significativas a um nível de 0,001 enquanto uma (o efeito da atratividade das alternativas sobre a lealdade) é significativa a um nível de 0,05.

Figura 2 - Coeficientes Padronizados Estimados para o Modelo Proposto

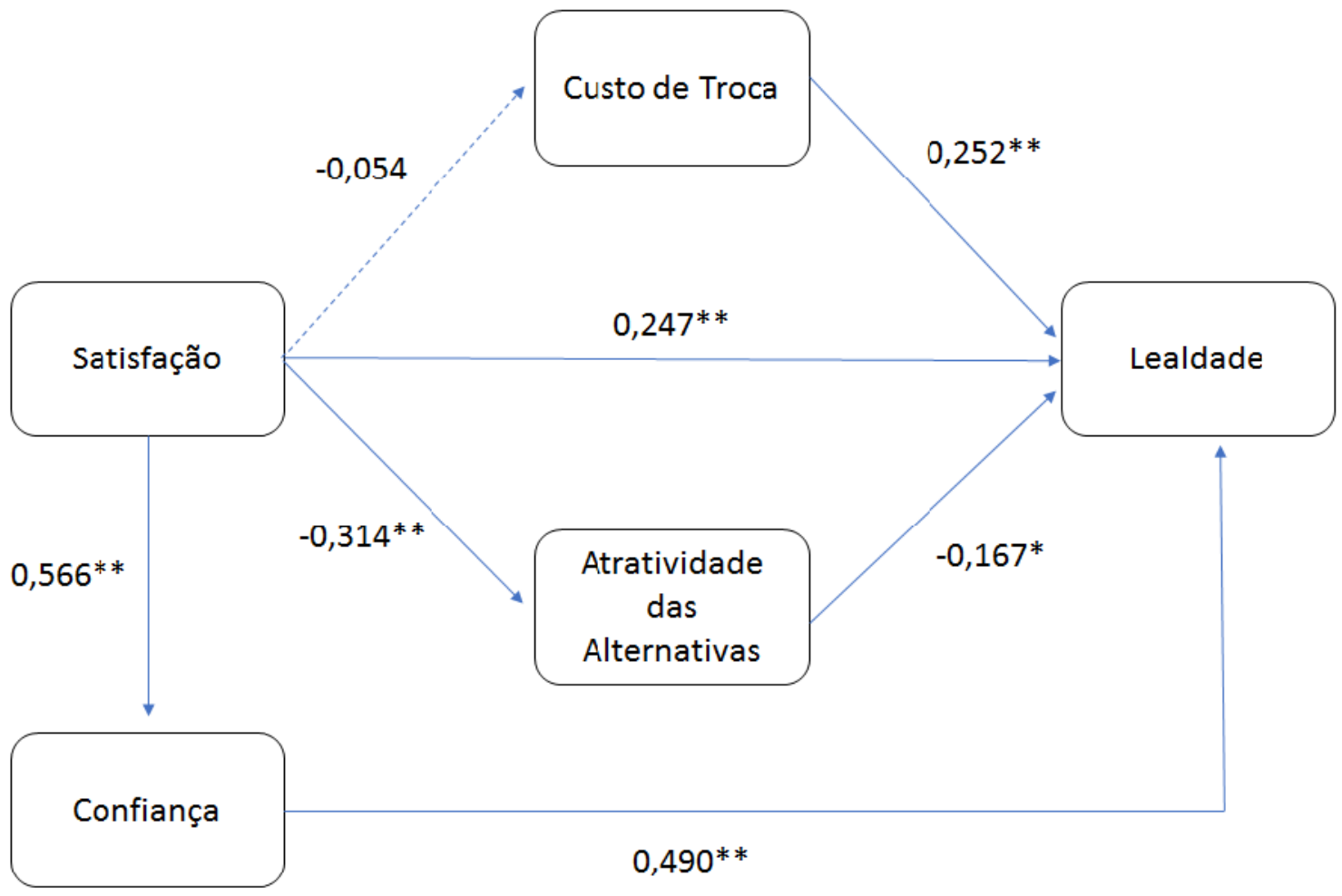

(* indica $p$-value $<0,05 ; * *$ indica $p$-value $<0,001$ )

Fonte: Elaborado pelos autores, 2019.

\section{DISCUSSÃO DOS RESULTADOS}

Esta seção discute os resultados obtidos, buscando cruzar com a literatura apresentada anteriormente com o objetivo de discutir cada hipótese do modelo. 


\subsection{SATISFAÇÃO}

A confirmação da relação entre a satisfação e a lealdade, hipótese H1, está de acordo com os resultados obtidos por outros pesquisadores (OLIVER, 1999; PATTERSON; SMITH, 2003; TSAI et al., 2006; VALVI; FRAGKOS, 2012). O resultado indica um efeito direto e razoavelmente significativo (coeficiente padronizado de 0,247 ) da satisfação sobre a lealdade do cliente no mercado de seguros. Este resultado reforça as conclusões do estudo de Picón et al. (2014), em que o autor sugere que as seguradoras devem focar na qualidade de serviço quando ofertam seus seguros.

A relação entre a satisfação e o custo de troca, representada pela hipótese $H 2$, não foi confirmada. A pesquisa apontou uma relação não significativa ( $p$-value de 0,447$)$ entre satisfação do cliente e custo de troca. O resultado não está de acordo com estudos anteriores (NGOBO, 2004; BARROSO; PICÓN, 2012; 53 PICÓN et al., 2014), que afirmam que a satisfação do cliente tem um efeito positivo sobre os custos de troca. O que pode ser uma explicação para o resultado encontrado é o fato de o mercado de seguros brasileiro apresentar muita concorrência. Atualmente mais de 10 seguradoras atuam com seguro de automóvel. A maioria investe na qualidade do serviço ao mesmo tempo que buscam oferecer seguros com preços competitivos, e as ofertas de produtos são relativamente semelhantes. Assim, a satisfação é uma exigência dos consumidores e não um diferencial, fazendo com que a satisfação não influencie diretamente no custo de mudança de seguradora. Outro fator a ser destacado é a rigorosa regulação da autarquia que supervisiona todo o mercado de seguros, que de certa forma padroniza os serviços das grandes companhias de seguro de automóvel. Como os segurados se sentem amparados pela fiscalização do governo e pelo código de defesa do consumidor, podem ter uma percepção menor de risco de ter uma experiência de consumo insatisfatória, fazendo com que a percepção do custo de mudança não seja fator relevante na decisão. Por fim, pode haver uma certa distância entre o segurado e a seguradora devido à relevância do papel do corretor, que ainda é muito forte na mediação entre as partes. O corretor é um intermediário que é responsável por representar o cliente perante a seguradora, diminuindo a percepção de perda no relacionamento em uma eventual troca de seguro. 
Com relação à H3, a influência da satisfação na atratividade das alternativas, essa hipótese foi verificada (coeficiente de -0,341). O resultado corrobora estudos anteriores (PATTERSON; SMITH, 2003; ANTÓN et al., 2007; PICÓN et al., 2014). A confirmação dessa hipótese sugere que quanto maior a satisfação de um cliente maior será a sua resistência a iniciativas de persuasão de concorrentes. O cliente satisfeito terá uma probabilidade maior de perceber a atratividade das alternativas como tendo nível mais baixo (BANSAL et al., 2005). Os segurados mais satisfeitos percebem como menor o benefício da troca, tendo em vista que possuem maior probabilidade de avaliar seu seguro de automóvel como superior na análise custo-benefício perante a concorrência. Assim, a satisfação atua diretamente na percepção de que outros seguros existentes no mercado são menos atraentes do que o atual. Outro motivo da satisfação possuir relação direta e significativa com a atratividade das alternativas, é que indivíduos quando insatisfeitos com aspectos como qualidade de serviço ou preço tendem a procurar conhecer alternativas disponíveis, apresentando, portanto, maior inclinação para o rompimento do relacionamento. Em contrapartida, a satisfação inibe a inclinação de mudança, diminuindo assim a atratividade das alternativas (ANTÓN et al., 2007).

\subsection{CUSTO DE TROCA, ATRATIVIDADE DAS ALTERNATIVAS E LEALDADE}

A relação entre custo de troca e lealdade dos clientes, hipótese H4, se mostrou significativa (coeficiente de 0,252). Esse resultado está alinhado com as conclusões de estudos anteriores (MORGAN; HUNT, 1994; NGOBO, 2004; BANSAL et al, 2005; PICÓN et al., 2014), que sugerem que há uma relação entre os dois construtos. A relação entre custo de troca e lealdade indica que quando os clientes entendem que o esforço para adquirir um seguro é grande, eles tendem a se manter leais. De acordo com a tipologia de custo de troca de Burnham et al. (2003), pode-se destacar na pesquisa os resultados de custo de avaliação, aprendizagem e de inicialização, já que $54 \%$ dos respondentes afirmaram que não dispõe de tempo para buscar informações completas para avaliar outras seguradoras e $60 \%$ dos respondentes consideram que é preciso de muito tempo e esforço para obter todas as informações necessárias para avaliar confortavelmente uma nova seguradora. Entretanto, com relação ao custo de perda de relacionamento pessoal, essa não se mostrou significativa, 
mostrando que $90 \%$ do total não concordaram que sentiriam falta do relacionamento com as pessoas da atual seguradora.

Em grande parte, isso se explica pela figura do corretor, que ainda exerce um papel relevante na intermediação entre segurado e seguradora, distanciando um pouco esse relacionamento. Com relação às perdas monetárias e de benefícios, apenas $30 \%$ discordaram da afirmação que é caro pagar por todos os custos referentes à troca de seguro. Portanto, o esforço e a falta de tempo para avaliar alternativas, aliado com a percepção de que existem muita burocracia envolvida na troca na seguradora, aumenta a probabilidade de o segurado manter o relacionamento com a seguradora atual. A maior parte das seguradoras exige o preenchimento de um formulário para a cotação de um seguro de automóvel. Muitas vezes até o código do chassi é solicitado. Outra burocracia é o preenchimento do QAR (questionário de avaliação do risco), em que uma série de perguntas são feitas e que não são necessárias na renovação com a mesma seguradora.

A relação negativa entre atratividade das alternativas e lealdade, hipótese $H 5$, foi confirmada, estando de acordo com o resultado obtido pelos autores Bansal et al. (2005), Hung et al. (2006), Picón et al. (2014), apesar de ter apresentado um coeficiente relativamente baixo $(-0,167)$. Apesar da baixa magnitude desse coeficiente, esse resultado pode sugerir que a atratividade das alternativas pode atuar de certa forma como um fator que motiva e favorece a mudança para uma alternativa concorrente. Quanto maior a atratividade das alternativas disponíveis menor será a lealdade da marca atual. Conforme Bansal et al. (2005), se o cliente perceber que os concorrentes existentes no mercado podem oferecer um produto ou serviço com um melhor custo-benefício, ele pode acabar terminando o relacionamento com a marca atual. No mercado de seguros nacional é comum diferentes seguradoras de automóvel precificarem um mesmo seguro com valores diferentes. Se um cliente verifica a existência de outras seguradoras mais atrativas, ele exibirá menor lealdade. Com maior atratividade das alternativas, ele pode se sentir inclinado a trocar de seguro.

\subsection{CONFIANÇA}


A relação entre satisfação e confiança, hipótese H6, foi suportada, se alinhando a estudos anteriores (CHAUDHURI; HOLBROOK, 2001; SIRDESHMUKH et al., 2002). A satisfação aumenta positivamente a confiança. Esta pesquisa aponta que a relação entre a satisfação e a confiança é a mais significativa, com o coeficiente padronizado de 0,566. Infere-se, então, que a seguradora deve atender a expectativa do cliente para aumentar sua satisfação, gerando maior confiança. Sirdeshmukh et al. (2002) sugerem que a confiança é baseada na percepção de que a empresa tem capacidade de desempenhar sua função declarada e é responsável pelo interesse e bem-estar do cliente. Dentro do seguro, clientes muitos satisfeitos podem ter confiança na dimensão da competência, se baseando na expectativa de um desempenho satisfatório da seguradora. Como uma apólice de seguro representa uma proteção financeira contra riscos futuros, é importante a confiança na formação de um relacionamento com uma seguradora. Ela deverá ser capaz de honrar seus compromissos em algum evento desfavorável. A satisfação no seguro também pode ocorrer na prestação de serviços emergenciais como reboque ou mecânico, que pode aumentar a percepção de benevolência, já que esse tipo de assistência pode trazer um bem-estar físico e psicológico. Por fim, como o contrato de seguro é pautado pela boa-fé, quando uma seguradora efetua um pagamento a uma indenização, o segurado pode ter maior confiança em sua dimensão da integridade (MAYER et al., 1995).

Por fim, a relação entre confiança e lealdade, hipótese H7, também foi confirmada e corrobora estudos anteriores (CHAUDHURI; HOLBROOK, 2001; SIRDESHMUKH et al., 2002; AURIER; N'GOALA, 2010), também apresentando uma magnitude relativamente forte com um coeficiente padronizado de 0,490 . O resultado para esta relação direta e positiva da confiança na lealdade é a de que o segurado se baseia na crença de um pagamento de uma indenização em caso de um eventual prejuízo. Desta forma, é natural que a confiança na seguradora seja um antecedente da lealdade do cliente de seguros de automóvel. Este resultado corrobora com o estudo de Sirdeshmukh et al. (2002), que sugere que a dimensão da competência é fundamental para o desenvolvimento da confiança e se baseia na expectativa de um desempenho satisfatório e qualificado por parte da seguradora. $\mathrm{Na}$ pesquisa, foi identificado que o fator mais importante na contratação de um seguro de automóvel para os respondentes é a credibilidade e a reputação da seguradora. Sendo que $61 \%$ dos respondentes afirmam acreditar que sua seguradora atual é integra. Ou seja, a 
pesquisa demostra a dimensão integridade da confiança como importante na construção de um relacionamento de longo prazo. O resultado está alinhado com outros estudos sobre a confiança (DWYER et al., 1987, PALMATIER et al., 2006), em que os autores afirmam que a confiança existe quando uma das partes acredita na integridade do parceiro de troca.

\section{CONSIDERAÇÕES FINAIS}

A principal contribuição deste trabalho é propor um novo modelo a partir de estudos anteriores (AURIER; N'GOALA, 2010; PICÓN et al., 2014), agregando um outro olhar sobre os construtos e a possibilidade de novas relações e interpretações. Dessa forma, os resultados aqui obtidos e as relações verificadas na pesquisa representam duas implicações para a teoria de comportamento do consumidor, aprofundando o conhecimento sobre a lealdade de clientes de seguros de automóvel.

Primeiro, confirma a importância da satisfação na lealdade, conforme apontado anteriormente por Dick e Basu (1994), Oliver (1997), Caruana (2002) e Picón et al. (2014) e sugere que a confiança é o construto com relação mais significativa na formação da lealdade, seguido pelo custo de troca e a atratividade das alternativas. A inclusão da confiança no modelo aumenta a compreensão de como o consumidor se comporta e como a sua lealdade e satisfação são construídas, principalmente no setor de seguros, na qual o benefício da utilização do produto, em caso de sinistro, somente ocorre em um período futuro. Assim, a confiança desempenha um papel importante, uma vez que o consumidor precisa confiar que a empresa irá desempenhar o que foi prometido, caso seja necessário.

Segundo os resultados da pesquisa sugerem que um cliente satisfeito percebe como menos atraente as alternativas de concorrentes, isto é, um cliente satisfeito é mais resistente às tentativas de persuasão da concorrência. Todavia, o resultado da pesquisa não pode confirmar que a satisfação aumenta o custo de troca percebido do segurado, dificultando a quebra do relacionamento do cliente com a seguradora atual. Por fim, a pesquisa apontou que a satisfação possui uma relação direta e muito significativa na confiança.

Quanto às implicações práticas, o resultado encontrado de que a satisfação influência fortemente a lealdade evidencia a importância da satisfação do cliente, Dessa forma, a Revista Eletrônica de Estratégia \& Negócios, Florianópolis, v.12, n. 3, set./dez. 2019. 
seguradora deve investir na qualidade do atendimento, no treinamento de seus colaboradores, na personalização de serviços e diversificação de coberturas que proporcionem uma apólice que atenda a todas as necessidades dos segurados. Buscando maior nível de satisfação, as seguradoras evitarão que seus clientes procurem e se interessem pela concorrência, diminuindo, assim, a atratividade das alternativas. Outra estratégia da seguradora poderá ser o desenvolvimento de tecnologias que aproximem o segurado, estreitando seu relacionamento que pode estar muito concentrado no papel do corretor, como os recentes aplicativos para celular.

Com relação ao custo de troca, muitos respondentes afirmaram que não concordam que exista muita perda financeira na troca de uma seguradora. Por lei, o cliente fica livre para trocar de seguradora a cada ano pela vigência da apólice. Nesses casos a seguradora deveria criar mecanismos de fidelização que aumentem a percepção de perda para o segurado, criando barreiras para o rompimento do relacionamento ou produtos relacionados, como oferta de cartão de crédito, vinculando o uso deste a futuros descontos no preço da apólice. Isso poderia aumentar o custo de mudança. Por fim, o fator considerado pelos respondentes como o mais importante na contratação de um seguro é a credibilidade da seguradora. Por isso é importante que as seguradoras se esforcem para o fortalecimento da marca, imprimindo uma imagem de integridade para seu cliente.

Quanto à limitação do estudo, no que diz respeito à coleta dos dados, apesar do instrumento de pesquisa ter sido aplicado somente para respondentes que tivessem conhecimento sobre seguros de automóvel, a falta de experiência ou conhecimento sobre o assunto pode ter influenciado a qualidade de algumas respostas. Para minimizar essa limitação da pesquisa, a aplicação do questionário poderia ocorrer após a renovação anual do seguro ou após o pagamento de alguma indenização. Entretanto, esse processo exigiria um acordo com as seguradoras, o que não foi viável para este estudo.

Como sugestão para pesquisas futuras, sugere-se a realização de estudos similares com uma amostra mais numerosa, e com perfil diferente do estudado neste trabalho, como, por exemplo, com clientes de outros estados e regiões do país, possibilitando, assim, uma forma interessante de validar e ampliar o escopo dos resultados obtidos. 
Em função das características e particularidades de cada tipo de seguro, aplicar o mesmo modelo com foco em outros ramos de seguros específico poderá gerar outras conclusões, e possivelmente implicações mais direcionadas e objetivas. Outras escalas e construtos também poderiam ser aplicados ao modelo, como por exemplo os antecedentes do custo de troca que podem explicar de que forma esse construto impacta na lealdade, ampliando a compreensão da formação da lealdade no mercado de seguros de automóvel. Outra sugestão é pesquisar o papel do corretor de seguro na lealdade de um cliente dentro de uma seguradora e avaliar os impactos desta intermediação na satisfação e na formação da lealdade do cliente junto à seguradora.

\section{REFERÊNCIAS}

AAKER, D. A.; KUMAR, V.; DAY, G. S. Marketing Research. Wiley, 9 Ed., 2006.

ANTÓN, C.; CAMARERO, C.; CARRERO, M. The Mediating Effect Of Satisfaction On Consumers' Switching Intention. Psychology And Marketing, v.24, n.6, p.511-538, 2007.

AURIER, P.; N'GOALA, G. The Differing And Mediating Roles Of Trust And Relationship Commitment In Service Relationship Maintenance And Development. Journal of the Academy of Marketing Science, v.38, n.3, p.303-325, 2010.

BANSAL, H. S.; TAYLOR, S. F.; ST. JAMES, Y. “Migrating” To New Service Providers: Toward a Unifying Framework of Consumers' Switching Behaviors. Academy Of Marketing Science Journal, v.33, n.1, p.96-115, 2005.

BARROSO, C.; PICÓN, A. Multi-Dimensional Analysis of Perceived Switching Costs. Industrial Marketing Management, v.41, n.3, p.531-543, 2012.

BELL, D. E. Regret in Decision Making Under Uncertainty. Operations Research, v.30, n.5, p.961-981, 1982.

BURNHAM, T. A.; FRELS, J. K.; MAHAJAN, V. Consumer Switching Costs: A Typology Antecedents, And Consequences. Journal of The Academy Of Marketing Science, v.31, n.2, p.109-126, 2003.

BYRNE, B. M. Structural Equation Modeling With Amos: Basic Concepts, Applications and Programming. 2a Ed. Routledge, NY, 2010.

CARUANA, A. Service Loyalty: The Effects of Service Quality and The Mediating Role Of Customer Satisfaction. European Journal of Marketing, v.36, n.7/8, p.811-828, 2002.

CHAUDHURI, A.; HOLBROOK, M. B. The Chain Of Effects From Brand Trust And Brand Affect To Brand Performance: The Role Of Brand Loyalty. Journal of Marketing, v.65, n.2, p.81-93, 2001. 
CHEN, M. F.; WANG, L. H. The Moderating Role of Switching Barriers on Customer Loyalty in the Life Insurance Industry. The Service Industries Journal, v.29, n.8, p.1105-1123, 2009.

CNSEG. Confederação Nacional das Empresas de Seguros Gerais, Previdência Privada e Vida, Saúde Suplementar e Capitalização. (2017). Estatística De Mercado. Rio De Janeiro 2016. Disponível Em: < Http://Www.Cnseg.Org.Br/Cnseg/Estatisticas/> Acesso Em: 11 De Março

CUNNINGHAM, R. M. Brand Loyalty - What, Where, How Much. Harvard Business Review, v.34, n.1, p.116-128, 1956

DAY, G. S. A Two-Dimensional Concept of Brand Loyalty. Journal of Advertising Research, v. 9, n. 3, p. 29-35, 1969.

DICK, A. S.; BASU, K. Customer Loyalty: Toward an Integrated Conceptual Framework. Journal of the Academy of Marketing Science. v.22, n.2, p.99-113, 1994.

DWYER, D.; SCHURR, P.; OH, S. Developing Buyer-Seller Relationships. Journal of Marketing, v.51, n.2, p.1-27, 1987.

FORNELL, C. National Customer Satisfaction Barometer: The Swedish Experience. Journal of Marketing, v.56, n.1, p.6-21, 1992.

FORNELL, C.; LARCKER, D. Evaluating Structural Equation Models with Unobservable Variables and Measurement Error. Journal of Marketing Research, v.18, n.1, p.39-50, 1981.

GARBARINO, E.; JOHNSON, M. The Different Roles of Satisfaction, Trust and Commitment for Relational and Transactional Consumers. Journal of Marketing, v.63, n.2, p.70-87, 1999.

GHAZALI, E.; NGUYEN, B.; MUTUM, D. S.; MOHD-ANY, A. A. Constructing online switching barriers: examining the effects of switching costs and alternative attractiveness on e-store loyalty in online pure-play retailers. Electronic Markets, v. 26, n.2, p. 157-17, 2016.

GREMLER, D. D.; BROWN, S. W. The Loyalty Ripple Effect: Appreciating the Full Value of Customers. International Journal of Service Industry Management. v.10, n.3, p.271- 291, 1999.

HAGHIGHI, M.; DOROSTI, A.; RAHNAMA, A.; HOSEINPOUR, A. Evaluation of Factors Affecting Customer Loyalty in Restaurant Industry. African Journal of Business Management, v.6, n.14, p.5039-5046, 2012.

HART, S.; SMITH, A.; SPARKS, L.; TZOKAS, N. Are Loyalty Schemes A Manifestation Of Relationship Marketing, Journal Of Marketing Management, v.15, n.6, p.541-562, 1999.

HAU, L. N,; THUY, P. N. Impact of Service Personal Values on Service Value and Customer Loyalty: A Cross-Service Industry Study. Service Business, v.6, n.2, p.137-155, 2012.

HU, L. T.; BENTLER, P. M. CUTOFF. Criteria for Fit Indexes in Covariance Structure Analysis: Conventional Criteria versus New Alternatives. Structural Equation Modeling, v.6, n.1, p.155, 1999. 
HUNG, J.; SILVERTHORNE, C.; CHEN, J. Organization Communication, Job Stress, Organizational Commitment, and Job Performance of Accounting Professionals in Taiwan and America, Leadership \& Organization Development Journal, v.27, n.4, p.242-249, 2006.

HUNT, H. K. Overview and Future Research Direction. In Conceptualization and Measurement of Consumer Satisfaction and Dissatisfaction. H. Keith Hunt, Ed. Cambridge, Ma: Marketing Science Institute. 1977

JACOBY, J.; KYNER, D. B. Brand Loyalty Vs. Repeat Purchasing Behavior. Journal of Marketing Research, v.10, feb. p.1-9, 1973.

KAHN, B. E.; KALWANI, M. U.; MORRISON, D. G. Measuring Variety Seeking and Reinforcement Behaviors Using Panel Data. Journal of Marketing Research, v. 23, n.2, p. 89100, 1986.

LOOMES, G.; SUGDEN, R. Regret Theory: An Alternative Theory of Rational Choice under Uncertainty. The Economic Journal, v.92, n.368, p.805-824, 1982.

MAYER, R. C.; DAVIS, J. H.; SCHOORMAN, F. D. An Integrative Model of Organizational Trust. Academy Of Management Review, v.20, n.3, p.709-734, 1995.

MORGAN, R.; HUNT, S. The Commitment-Trust Theory of Relationship Marketing, Journal of Marketing, v.58, n.3, p.20-38, 1994.

NESSET, E.; HELGESEN, $\varnothing$. Effects Of Switching Costs On Customer Attitude Loyalty To An Airport In A Multi-Airport Region. Transportation Research Part A: Policy and Practice. v.67, September, p. 240-253, 2014.

NGOBO, P. V. Drivers of Customers' Cross-Buying Intentions. European Journal of Marketing, v.38, n.9/10, p.1129-1157, 2004.

OLIVER, R. L. Satisfaction, a Behavioral Perspective on Consumers. London, Maidenhead, 1997.

OLIVER, R. L. Whence Customer Loyalty? Journal of Marketing, v.63, Special Issue, p.33-44, 1999.

PALMATIER, R. W.; DANT, R. P.; GREWAL, D.; EVAN, K. R. Factors Influencing The Effectiveness Of Relationship Marketing: A Meta-Analysis. Journal Of Marketing, v.70, n.4, p.136-153, 2006.

PARASURAMAN, A.; GREWAL, D.; KRISHNAN, R. Marketing Research. 2. Ed., South-Western College Pub, 2006.

PATTERSON, P. G.; E SMITH, T. A. Cross-Cultural Study Of Switching Barriers And Propensity To Stay With Service Providers. Journal Of Retailing, v.79, n.2, p.107-120, 2003. 
PEREIRA, H. G.; SALGUEIRO, M. F.; RITA, P. Online Purchase Determinants Of Loyalty: The Mediating Effect Of Satisfaction In Tourism. Journal of Retailing and Consumer Services, v.30, p. 279-291, 2016.

PICÓN, A.; RUIZ, C.; CASTRO, I. The Relationship Between Satisfaction And Loyalty: A Mediator Analysis. Journal Of Business Research, v.67, n.5, p.746-751, 2014.

PORTER, M. E. Competitive Strategy: Techniques For Analyzing Industries And Competitors, New York: The Free Press, 1980.

SHARMA, N. The Role of Pure and Quasi-Moderators in Services: An Empirical Investigation of Ongoing Customer-Service-Provider Relationships. Journal of Retailing and Consumer Services, v.10, n.4, p.253-262, 2003.

SIRDESHMUKH, D.; SINGH, J.; SABOL, B. Consumer Trust, Value, and Loyalty in Relational Exchanges. Journal of Marketing, v. 66, n. 1, p. 15-37, 2002.

SPERBER, A. D. Translation and Validation of Study Instrument for Cross-Cultural Research. Gastroenterology, v. 126, n. 1, p. 124-128, 2004.

SULTAN, P.; WONG, H. Y. Antecedents and Consequences of Service Quality in a Higher Education Context: A Qualitative Research Approach. Quality Assurance In Education, v.21, n.1, p.70-95, 2013.

TSAI, H.; HUANG, H.; JAW, Y.; CHEN, W. Why On-Line Customers Remain With A Particular ERetailer: An Integrative Model And Empirical Evidence. Psychology and Marketing, v.23, n.5, p.447-464, 2006.

TUCKER, W. T. The Development of Brand Loyalty. Journal of Marketing Research, v.1, n.3, p.32-35, 1964.

UNCLES, M. D.; DOWLING, G. R.; HAMMOND, K. (2003). Customer Loyalty And Customer Loyalty Programs. Journal of Consumer Marketing, v.20, n.4, p.294-316, 2003.

VALVI, A. C.; FRAGKOS, K. C. Critical Review of the E-Loyalty Literature: A Purchase-Centred Framework. Electronic Commerce Research. v.12, n.3, p.331-378, 2012.

WANG, Y. S.; TANG, T. I.; TANG,; J. T. (2001). An Instrument For Measuring Customer Satisfaction Toward Web Sites That Market Digital Products And Services. Journal of Electronic Commerce Research, v.2, n.3, p.89-102, 2001. 